\title{
Investigación del trabajo con el diario en el aula de estudiantes y profesores
}

\author{
Lucia Castellón ${ }^{1}$, Catalina Mujica ${ }^{2}$
}

\begin{abstract}
Resumen
Un estudio para evaluar el aporte realizado por el Programa Prensa y Educación, que dirige la Universidad Diego Portales, al ámbito escolar nos advierte los beneficios que puede tener incorporar el trabajo con los medios de comunicación a las prácticas pedagógicas. Según la percepción que los niños y los profesores participantes en el Programa entregaron a partir del estudio, podemos destacar que hay consenso para afirmar que este tipo de iniciativas contribuye a familiarizar a los estudiantes con el diario, a valorar el valor informativo de las noticias, a jerarquizar la información y a innovar en estrategias de enseñanza-aprendizaje, entre otros logros. Pero además se alcanzan logros no esperados, tales como motivar la expresión de opiniones por parte de los niños y contribuir en la formación de su conciencia ciudadana.
\end{abstract}

Palabras claves: educación, comunicación, diarios

\section{Abstract}

A study to assess the contribution made by the Press, and Education Program to the school sphere tells us about the benefits of including the work with the media into the teaching practices can have. According to the perception children, and participating teachers in the Program gave from the study, we can emphasize that there is agreement to state that this kind of iniciatives contributes, including, but not limited to, to familiarize the students with the newspaper, to value the information value of the news, to arrange information in order of importance, and to innovate in strategies of teaching-learning. But there also are unexpected goals that get achieved, such as motivating the opinions by the children, and contribuiting into the formation of their city life conscience.

Key words: education, communication, newspaper

T-Lucia Castellon Aguayo, periodista, profesora, creadora del programa Prensa y Educación, ex decana de la facultad de Comunicación de la Universidad Diego Portales. Actualmente es directora de post grados enla Facultad de Comunicación y Diseño de la Universidad Mayor en Sanliago de Chile.

2. Catalina Mujica Vicuña, Periodista, Licenciada on Comunicación Social y Magister(c) en Comunicación se desempeña en la Universidad Diego Portales como académica y en el diario El Mercurio como asesora educativa del Programa "El Mercurio de los Estudiantes". 


\section{Prensa y Educación: El diario como una ventana al mundo}

Decir que los medios de comunicación son una ventana al mundo, es tal vez uno de los clisés o lugares comunes más propios de los medios de comunicación y del periodismo en general.

Sin embargo, ésa es una de las principales conclusiones a las que hemos llegado a través de la aplicación del Programa Prensa y Educación. El programa consiste en trabajar con el diario en el aula, en las asignaturas propias del plan de estudio, con más de veinte diarios en todo el país y de haber trabajado con más de quinientas escuelas

El programa nació en 1988 con el fin de fomentar el hábito de lectura en los niños y formarlos como lectores de prensa, potenciando un consumo medial de mayor calidad en cuanto al tipo de información periodística recibida. Desde esa fecha hasta hoy se han capacitado más de cinco mil profesores de 570 colegios del pais, quienes ven en el diario una herramienta más del proceso pedagógico. Trabajamos con 180 mil alumnos de Enseñanza Básica y Media al año y, en el tiempo que lleva aplicándose Prensa y Educación, hemos formado a cerca de un millón de niños.

Es así como el diario ha sido utilizado como material didáctico en distintas asignaturas del curriculum, tales como Castellano, Estudio y Comprensión de la Naturaleza, Matemáticas, Ciencias Sociales y Artes Plásticas.

\section{La investigación}

La capacidad que tienen los diarios para insertar a los niños dentro de su entorno, a través de la apropiación educativa de este medio, es uno de los principales descubrimientos de la investigación que se realizó en el año 1999.

Entre los principales objetivos que tuvo el estudio se encontraban el describir la motivación que tienen los alumnos y profesores para trabajar con el diario en la sala de clases; determinar si a través de la participación en Prensa y Educación se incrementa el conocimiento e interés de los alumnos sobre la realidad nacional; y, conocer la percepción de los niños sobre el diario, después de haber participado en el programa.

Desde un punto de vista metodológico, la investigación tuvo un carácter descriptivo y se centró tanto en los aspectos cualitativos, como cuantitativos del fenómeno. De esta manera se esperaba poder profundizar en los distintos hallazgos que se fueran produciendo a partir de la investigación cuantitativa.

A partir de ello se realizaron encuestas, grupos focales y entrevistas en profundidad. Conforme a lo anterior, lo que primero se aplicó fue la encuesta, para detectar las principales variables que estaban en juego. So- 
bre la base de esos resultados, se realizaron los grupos focales y las entrevistas.

Cabe señalar que debido al alto número de niños y profesores que participaban en el programa, fue necesario extraer muestras para poder realizar las encuestas, tanto en los alumnos como en los docentes. En ambos casos, se realizaron muestreos aleatorios simples.

En el caso de los niños la encuesta fue aplicada a alumnos de $4^{\circ}, 5^{\circ}$ y $8^{\circ}$ Básico, pertenecientes a los colegios que participan en el programa. El instrumento constaba de preguntas cerradas, diferenciales semánticos y preguntas abiertas.

Además, se realizaron un total de 12 grupos focales para recabar más información de tipo cualitativa. Cada uno de ellos estuvo compuesto por seis niños $y$ fueron realizados en sus propios establecimientos educacionales.

A su vez, a los profesores también se ics aplicó una encuesta en un primer momento, para después realizar entrevistas en profundidad. Con todo esto se recopiló información sobre los distintos actores que están involucrados en el programa y se identificaron los principales logros.

\section{Los resultados}

Si bien es cierto que el Programa Prensa y Educación está orientado hacia el área educativa, la investigación demuestra que muchos de los resultados obtenidos trascienden lo académico y se instalan dentro de ámbitos tan importantes como la participación ciudadana y la comprensión del mundo que los rodea.

Es así como los logros obtenidos pueden dividirse en cuatro puntos fundamentales, tales como la percepción sobre el diario como medio de comunicación, la familiarización con el diario, las contribuciones al ámbito educativo y el fomento a la lectura.

Con respecto al primero de los puntos, puede observarse un gran cambio después de la aplicación del programa. Antes de participar en Prensa y Educación, el 39,9\% de los niños veía a los diarios como algo aburrido y que sólo era para los adultos. Después de participar en el programa, la percepción negativa bajó a sólo un $6,5 \%$.

Asimismo el $90,6 \%$ manifestó que los diarios eran para los niños y los adultos, dado que ambos grupos debian estar informados. Es necesario hacer notar que cn los grupos focales los principales atributos con los que relacionaron a este medio de comunicación fueron: "informa", "entretiene", "enseña" y "es bueno".

Al profundizar en dichos calificativos se puede advertir que al diario lo encuentran "informativo", porque les sirve para enterarse de los hechos que ocurren en el pais y en el extranjero. Además les permiten interiorizarse 
de los asuntos que a ellos les interesan, como deportes y espectáculos.

En cuanto a la entretención, en la mayoría de los casos lo relacionaron con la posibilidad de informarse sobre "cosas que pasan" y de involucrarse en temáticas nuevas para ellos, como la política y los acontecimientos nacionales e internacionales. Llama la atención que la entretención fuera asociada a la adquisición de conocimientos sobre nuevas realidades para ellos y no, por la inclusión de materiales lúdicos, tales como historietas, puzzles y suplementos magazinescos.

Otro punto interesante del focus group, es que los alumnos dijeron que ellos aprenden en la medida en que leen noticias de actualidad. En este sentido, el carácter informativo de los periódicos lo relacionan directamente con la dimensión educativa.

Al participar en el programa los niños no sólo cambiaron su percepción sobre los diarios, sino que valoraron en gran medida el factor informativo, el que vincularon con dos aspectos que normalmente se ven contrapuestos, como los son la entretención y el aprendizaje.

Para todas las personas que formen parte del mundo de la educación a nivel escolar o universitaria, lograr que las clases sean entretenidas para los alumnos es uno de los desafíos más importantes, debido a que la motivación es uno de los factores que pueden determinar el éxito o el fracaso de un proceso educativo. Es por eso que la entretención no puede ser dejada de lado dentro de la educación.

Pero lo que más llama la atención es que según los niños al leer temas de actualidad se está aprendiendo. $Y$ es aún más llamativo que encuentren entretenido enterarse de hechos y acontecimientos de carácter político e internacional, si consideramos que vivimos en un pais en donde la inscripción en los registros electorales por parte de los jóvenes es cada vez más escasa..

El hecho de que un niño de sexto básico diga que el diario "es entretenido porque uno sabe de política, internacional y el tiempo", debe hacernos reflexionar más al respecto. Además esto se ve reforzado al analizar el tipo de noticias que les gustan. Si bien es cierto que deportes y espectáculos siguen manteniendo los primeros lugares, el ámbito policial, nacional, científico e internacional, tienen un alto grado de preferencias.

Estamos conscientes que sería bastante aventurado decir que la utilización del diario en las salas de clases resolvería el problema de la participación ciudadana. Sin embargo, los datos demuestran que ésta es una realidad que no puede ser pasada por alto.

$\mathrm{Y}$ tal como lo veremos más adelante, el factor educativo que se introduce con el programa va más allá de la formación de nuevos lectores de medios de comunicación, ya que sus resultados se escapan de la mera alfabetización medial. Por lo pronto, observamos que el diario permite unir la entretención con el ámbito educativo, debido a que abre las puertas hacia 
mundos que antes les eran desconocidos, como los son la política y la actividad cientifica.

\section{La familiarización con el diario}

Dentro de la encuesta efectuada a los profesores, uno de los aspectos que entregó un mayor número de hallazgos interesantes fue el grado de familiarización con el diario que los niños experimentaron después de participar en el programa.

Junto con conocer más de cerca este medio de comunicación y saber cuáles son las distintas secciones que tiene, se observó una mayor comprensión de lectura asociada a las noticias de los diarios.

Es así como el $84 \%$ de los docentes encuestados asegura advertir algún cambio en la disposición de los alumnos hacia el diario, después de participar en el programa.

Profesores: Desde que trabajan con el diario, los alumnos han aprendido a

\begin{tabular}{|l|c|}
\hline Buscar noticias dentro del diario & $93 \%$ \\
\hline Identificar las secciones del diario & $84 \%$ \\
\hline $\begin{array}{l}\text { Diferenciar entre una noticia informativa y } \\
\text { una de opinión }\end{array}$ & $50 \%$ \\
\hline Conocer la estructura de la noticia & $38.6 \%$ \\
\hline $\begin{array}{l}\text { ldentificar las diferencias entre la redacción } \\
\text { de un texto narrativo y un texto noticioso }\end{array}$ & $36.3 \%$ \\
\hline $\begin{array}{l}\text { Diferenciar los distintos géneros } \\
\text { periodisticos }\end{array}$ & $36.3 \%$ \\
\hline
\end{tabular}

Asimismo los principales aprendizajes logrados por los alumnos, según los profesores, fueron: buscar las noticias dentro del diario (93\%), identificar las secciones (84\%) y diferenciar entre una noticia informativa y una de opinión ( $50 \%$ ). Lo que muestran esos datos es que los niños se familiarizaron en un alto grado con el diario. Eso se ve reflejado en que no sólo identificaron las distintas secciones, sino que también fueron capaces tomar una noticia y categorizarla según los propios esquemas de los medios de comunicación, para después ir a buscarla alli. A lo que nos referimos es que al decir que los niños fueron capaces de buscar noticias dentro del diario, lo que hicieron fue manifestar que una noticia determinada correspondia a nacional o policial y después ir a buscarla dentro del diario.

Además es destacable que en un alto porcentaje los alumnos 
lograran ciertos conocimientos que son propios de la profesión de un periodista, como diferenciar entre una nota informativa y una de opinión, conocer la estructura de la noticia e identificar las diferencias entre la redacción de un texto narrativo y un texto noticioso.

Lo más interesante es que de acuerdo al $61.3 \%$ de los profesores, los alumnos demostraron un alto interés por conocer las noticias, después de participar en el programa. Durante los grupos focales uno de los docentes afirmó que a los niños "les gusta leer, comentar las noticias y se saben más conocedores de lo que pasa en el mundo. Relacionan noticias de la TV con el diario".

Dicha opinión, además de ser representativa de lo que sucedió durante los grupos focales, apunta también a otras direcciones. La primera de ellas es que a través del Programa Prensa y Educación se logró un cambio de hábitos, al crear en los alumnos la necesidad de estar informados.

A futuro esos niños no sólo van engrosar el número de lectores de los diarios, sino que deberían tener una menor dificultad para insertarse en el mundo, debido a que poseerán una mayor habilidad para leer el contexto en el que se mueven.

Eso se debe a que a partir de los grupos focales realizados con los alumnos se puede inferir que ellos saben distinguir entre lo que es importante o no, según el grado de relevancia que tiene para la comunidad. De hecho, al pedirles que ordenaran según el grado de importancia una serie 44 de noticias, primero pusieron artículos de "nacional" y después, de "política". Al preguntarles por qué habían establecido ese orden, dijeron que esas eran las noticias "más serias".

Esto que, a primera vista puede ser poco relevante, adquiere real sentido dentro del contexto de la llamada Sociedad de la Información. En este mundo de Internet y de la abundancia de la información, es vital poder distinguir entre lo importante y lo accesorio. Si hasta los años ochenta el secreto era la principal herramienta para ejercer la desinformación, en este momento el exceso de información cumple el mismo papel.

Un claro ejemplo fue la Guerra del Golfo, en donde pese a la cobertura tipo CNN de 24 horas al dia, supimos menos que nunca. Incluso, lo mismo podría aplicarse a lo que está sucediendo en este minuto con los acontecimientos posteriores a los ataques terroristas del 11 de septiembre de este año.

Además esta capacidad de jerarquización está estrechamente relacionada, con un aumento en la comprensión de lectura. El $77.2 \%$ de los profesores encuestados dijo que los niños han logrado comprender mejor las noticias que aparecen en los diarios.

Durante los grupos focales uno de los alumnos de sexto básico aseguró que las noticias "las entiendo más porque ahora uno puede relacionarlas". A partir de todo esto puede aventurarse la hipótesis de que 
los diarios lo que han realizado es abrir una ventana hacia el mundo. Junto con mostrarles lo que ocurre en él, les ha permitido entender, relacionar y jerarquizar los distintos acontecimientos.

Como podemos observar éste es un campo que trasciende al ámbito educativo, pero que es de vital importancia para que en el futuro cercano los alumnos sean capaces de insertarse en la denominada Sociedad de la Información.

\section{Las contribuciones del diario al ámbito educativo}

Si bien es cierto que hasta el momento nos hemos centrado en aquellos aspectos que se ubican fuera de lo educativo, uno de los principales descubrimientos de la investigación es la manera en cómo el diario se ha transformado en una herramienta pedagógica.

El $97.7 \%$ de los docentes entrevistados afirmó que el diario sirve para utilizarse en la sala de clases, tal como lo es un libro y otro material de la misma especie. Asimismo el $97 \%$ de los profesores estima que el Programa Prensa y Educación ha sido un aporte para el desarrollo de su experiencia pedagógica.

Además el $90.9 \%$ de los profesores expresó que la incorporación del diario a la sala de clases les ayuda en el logro de los objetivos de la asignatura. Por otra parte, el $90 \%$ estuvo de acuerdo con la afirmación de que el programa les permite hacer clases más participativas. Y el $88.6 \%$ dijo que los alumnos se motivan más para trabajar en clases, lo que está en estrecha relación con la afirmación de que el $77.2 \%$ de los maestros manifiesta que Prensa y Educación les facilita su labor docente.

Sin embargo, los profesores están conscientes de que el diario puede llegar a ser educativo en la medida que ellos mismos le den esta orientación, transformándolo en una herramienta que les permita innovar las estrategias de aprendizaje.

Al respecto, uno de los maestros opinó durante un grupo focal que "el diario es un elemento que podemos encontrar tan a la mano y se puede utilizar fácilmente, pero no para salvar el momento, sino que para darle un sentido al trabajo con él".

Eso refuerza la idea de que el diario puede convertirse en una herramienta pedagógica y que con él pueden lograrse objetivos importantes, al hacer más participativas las clases y mejorar la motivación de los alumnos. 


\section{Profesores: Principales aportes del Programa Prensa y Educación}

\begin{tabular}{|l|c|}
\hline $\begin{array}{l}\text { Ayuda en el logro de objetivos } \\
\text { pedagógicos }\end{array}$ & $91 \%$ \\
\hline $\begin{array}{l}\text { Me permite hacer clases más } \\
\text { participativas }\end{array}$ & $90 \%$ \\
\hline Tengo acceso gratuito al material & $88.6 \%$ \\
\hline Los alumnos se motivan más & $88 \%$ \\
\hline Coincide con los objetivos de la Reforma & $79.5 \%$ \\
\hline Me facilita mi labor docente & $77.2 \%$ \\
\hline $\begin{array}{l}\text { Me permite trabajar ciertos objetivos } \\
\text { mejor que con cualquier otro material }\end{array}$ & $59 \%$ \\
\hline
\end{tabular}

Como resultado de ello el $91 \%$ de los docentes asegura que las clases fueron más entretenidas, tanto para ellos como para los niños. Los alumnos, por su parte, explicaron durante los grupos focales que se entretienen porque el ambiente de la clase se torna más relajado. Si bien se produce desorden, es un desorden organizado que no impide que realicen las actividades que les propone el profesor.

A través de los focus group se descubrió que los profesores estimaban que este fenómeno se generaba porque los alumnos al trabajar con el diario, tenían la posibilidad de escoger las informaciones con la iban a trabajar, de acuerdo con sus propios intereses.

A partir de allí también se explica el hecho de que el $88.1 \%$ de los profesores encuestados estime que con el programa los alumnos se convierten en protagonistas de su aprendizaje, alcanzando un mayor grado de autonomía.

Por lo tanto, el diario se convierte en un elemento motivador debido a que logra generar un cambio metodológico al interior de la sala de clases. $Y$ este cambio se centra en el hecho de que se alcanza un cierto grado de flexibilidad en su utilización, al permitir que el alumno escoja entre una amplia gama temática, de acuerdo a sus intereses o a las ganas de introducirse en realidades que son nuevas para él.

\section{El fomento a la lectura}

Entre los mayores logros del trabajo realizado por el Programa Prensa y Educación, a juicio de los docentes, se encuentra el desarrollo de la expresión, de la comunicación y la motivación por la lectura. 
El $72.2 \%$ de los profesores asegura que trabajar con el diario ha sido una valiosa herramienta para motivar a los alumnos en la lectura de una manera dinámica y entretenida. Explican que los alumnos se interesan en leer de manera espontánea, tanto porque pueden escoger textos de su interés, como porque comienzan a encontrarle un sentido práctico a esta lectura: obtener información.

Si bien no es posible ver si existe un aumento concreto de la lectura, dado que ese es un proceso a largo plazo, se puede advertir altos porcentajes de lectura del diario por parte de los alumnos. Un $50 \%$ de los niños afirma que lee el diario en otros momentos, a parte de cuando el profesor se los pide en clases y un 30\% dice que lo hace a veces.

Mientras que $16 \%$ dice que lee el diario cuando está aburrido. Llama la atención el alto porcentaje de alumnos de $4^{\circ}$ básico que tiene esa conducta, debido a que es el mismo grupo que más lee el periódico aparte de la sala de clases.

Otro aspecto interesante es que el $93 \%$ de los profesores opina que la habilidad para formular opiniones propias es la competencia que más se desarrolló con el diario. Y lo más interesante, es que el $88.5 \%$ asegura que los alumnos desarrollaron opiniones sobre los hechos de actualidad.

\section{Conclusión}

Sin lugar a dudas, el hecho de los niños formulen opiniones propias sobre las distintas noticias, es uno de los principales logros de Prensa y Educación, ya que en él se resumen todos nuestros objetivos como programa.

No sólo se aporta a la educación de los alumnos de la Enseñanza Básica y Media, sino que también se abre su mente al mundo al relacionarlos con su contexto inmediato. Recordemos que desde un punto de vista deontológico, ése es uno de los principales deberes del periodismo, debido a que se enmarca dentro de la esencia del derecho a la información.

Además al entregarle herramientas a los niños para que puedan jerarquizar las informaciones y separar entre lo importante y lo accesorio, se los está preparando para vida en la Sociedad de la Información. Al tener esas habilidades podrán navegar entre estos mares de información que lejos de comunicar y enriquecer, terminan por desorientar y desinformar a la población.

Por otra parte, la educación medial surge como una alternativa válida para fomentar la participación politica por parte de los jóvenes. Si no se logra que se interesen por la politica desde niños 0 , lo que es peor, nunca han lefdo nada al respecto, es muy dificil poder convencerlos cuando cumplan 18 afios. 
Como podemos observar, el Programa Prensa y Educación, al promover la utilización del diario en la sala de clases, ha logrado una serie de resultados que van más allá de lo educativo y del fomento a la lectura, lo que nos llama a seguir trabajando.

\section{Referencias bibliográficas}

Aparici, Roberto. La enseñanza de los medios. 1995.Revista Cuadernos de Pedagogía, No. 241.Editorial Fontalba, España

Avendaño, Claudio. Proyecto Prensa y Educación. 1996. En Encuentro 'Voces que se unen'. Ministerio de Educación; Universidad Diego Portales. Santiago, Chile. pp 30-41

Cardemil, Cecilia. Comunicaciones que construyen una disciplina eficiente en la sala de clases. 1989. En García Huidobro, J. E. Escuela, Calidad e Igualdad. Los desafios para la educación en democracia. CIDE, Santiago. pp 173-218

Coll, Cesar. Significado y sentido en el aprendizaje escolar. Reflexiones en torno al concepto de aprendizaje significativo. Revista Infancia y Aprendizaje, No. 41. Barcelona. http://www.educadormarista.com/Descognitivo/ significado_y_sentido.htm

Concha, Carlos. Reforma Educacional y cambio pedagógico. 1997. Documento de la División de Educación General, Ministerio de Educación. 6 pag.

Condemarín, Galdames, Medina. Lenguaje integrado. 1992. Mineduc, CPEIP, Santiago, Chile, pags. 10-11

Condemarín, M, Galdames, V y Medina, A. ¿Cómo aprenden los niños?: Implicaciones para el desarrollo de estrategias pedagógicas en el aula. 1994. Documento de Trabajo para las Jornadas P-900.

Kaplún, Mario. A la Educación por la comunicación. La práctica de la comunicación educativa. 1992. Primera Edición. UNESCO/Orealc. Santiago, Chile. 234 pag.

Monsalva Mena, Sergio. Educación: comunicación, psicologia e integración. 1994. Documentos de Trabajo $n^{\circ} 7$. Centro de Estudios y Atención del Niño y la Mujer (CEAMIN). Santiago, Chile. 21 pag.

Montalbán, Manuel Vázquez. La formación de la conciencia. Copyright 2000 Quaderns Digitals numero 21. Comunicación de masas, lenguaje y educación abril junio 1997 
Mujica, Catalina. Percepción de los alumnos y profesores del Programa La Tercera en Mi colegio. 1999. Santiago, Chile.

Ottone, Ernesto. Modernos y ciudadanos a la vez. Notas acerca de la legalización y la transformación educativa. 1997. Docencia. Revista del colegio de profesores de Chile A.G. Año 2, $n^{\circ}$ 2. Santiago, Chile.pp 14-21

Pinto, Rolando. Una mirada crítica a los fundamentos históricos y situacionales de la Educación chilena, en los años 90. 1997. Docencia. Revista del colegio de profesores de Chile A.G. Año 2, $n^{\circ}$ 2. Santiago, Chile. pp 5-13.

Rodrigo, Alsina Miquel. Por un uso critico de la prensa. La construcción periodística de la realidad social. Signos Teoría y práctica de la educación, 21 Abril - Junio de 1997 Páginas 20-24

Schiefelbein, Ernesto. En búsqueda de la nueva del siglo XX. 1992. Primera Edición. Santiago, Chile. Talleres de Corporación de Promoción Universitaria. 150 pág.

Simpson, Michelle y Nist, Sherrie. Actualización sobre el aprendizaje estratégico: va mas allá de las estrategias para leer textos. Revista Journal Adolescent \& Adult Licteracy. Marzo 2000.

Solé, Isabel. Para comprender antes de la lectura. 1996 Estrategias de Lectura. Editorial Graó. Barcelona, España.

Vygotsky, Lev. Pensamiento y lenguaje. 1977. Editorial La Pléyade. Buenos Aires. Argentina. 


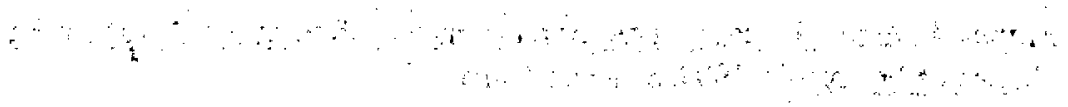

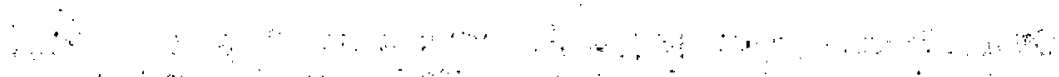

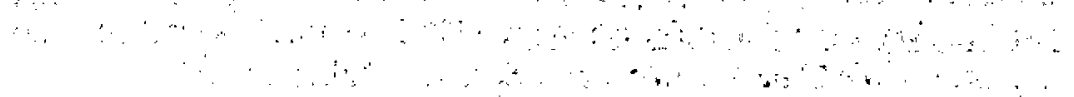

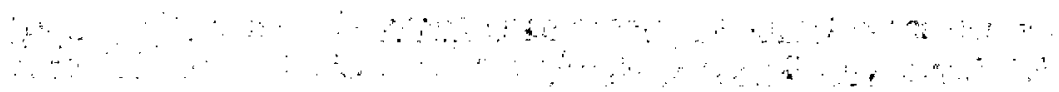

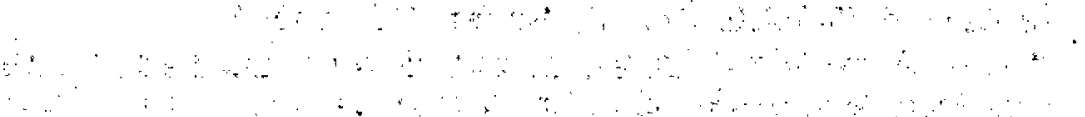

$$
\begin{aligned}
& \therefore \quad \therefore \quad \therefore \quad \therefore
\end{aligned}
$$

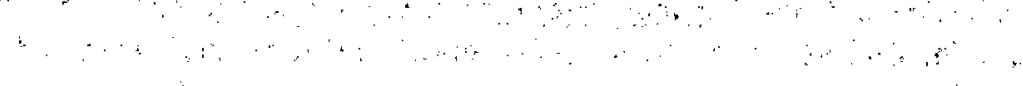

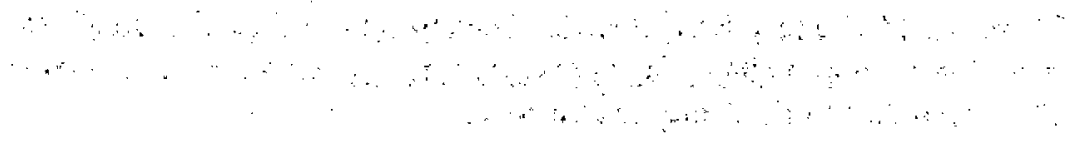

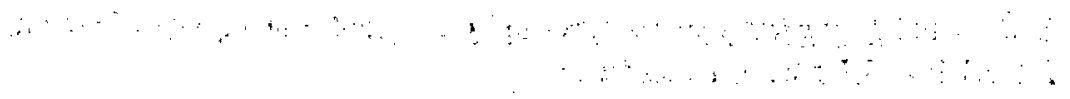

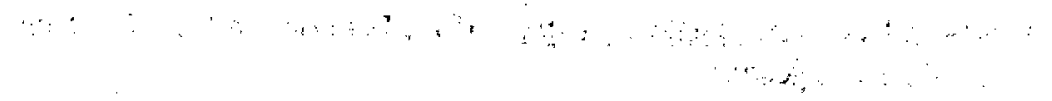

